\title{
Avaliação do Coeficiente de Difusão de Cloretos como Parâmetro de Durabilidade em Concretos com Diferentes Classes de Resistência Mecânica ${ }^{1}$ e sem Adições Minerais
}

\author{
R. C. LOPES ${ }^{1}$, A. M. OLIVEIRA ${ }^{1 *}$, O. CASCUDO ${ }^{1}$ \\ *Autor de Contacto: andriellimorais@ufg.br \\ ${ }^{1}$ GEDur - Grupo de Estudo em Durabilidade, Laboratório de Inovação Tecnológica em Construção Civil - \\ LABITECC, PPGGECON - Programa de Pós-Graduação em Geotecnia, Estruturas e Construção, Escola de \\ Engenharia Civil e Ambiental, Universidade Federal de Goiás - UFG, 74.605-220. Praça Universitária, Goiânia - \\ GO,Brasil, clopesrayane@gmail.com, andriellimorais@ufg.br*, ocascudo@ufg.br
}

\begin{abstract}
RESUMO
Parâmetros de durabilidade podem ser ferramentas úteis e interessantes em uma abordagem baseada na ciência de dados e na modelagem da performance em campo de estruturas em concreto armado. $\mathrm{O}$ coeficiente de migração de cloretos em estado não estacionário - $\mathrm{D}_{\text {nssm, }}$, (coeficiente aparente), obtido pelo método NT Build 492, pode ser um marcador de durabilidade do concreto, ao mesmo tempo em que, é um ensaio de simples execução em laboratório e é uma informação de entrada em modelos matemáticos de predição de vida útil. Assim, é objetivo deste trabalho analisar a sensibilidade do $D_{\text {nssm }}$ em concretos sem adições minerais e com a interveniência de três classes de resistência (MPa), a saber $\mathrm{C} 45, \mathrm{C} 30$ e C25, as quais correspondem as relações água/cimento (a/c) 0,$40 ; 0,55$ e 0,70 , respectivamente. Como resultado esperado, houve correlação $\left(R^{2}=0,99\right)$ entre o parâmetro resposta $D_{\text {nssm }}$ e a variável relação a/c ou classe de resistência nos níveis estudados. Com a alteração da relação a/c de 0,70 para 0,55 de 0,70 para $0,40, D_{\text {nssm }}$ reduziu em $41 \%$ e $61 \%$, respectivamente. Os resultados foram discutidos com base na microestrutura dos concretos, análise estatística e no coeficiente de migração, como um parâmetro de durabilidade.
\end{abstract}

Palavras chave: Cloretos, migração, classe de resistência, NT Build 492, parâmetro de durabilidade.

\begin{abstract}
Durability parameters can be useful and interesting tools in a data science-based approach and in

\footnotetext{
${ }^{1}$ Associação Brasileira de Normas Técnicas. (2015). NBR 8953: Concreto para fins estruturais - Classificação pela massa especifica, por grupos de resistência e consistência. Rio de Janeiro.
} 
field performance modeling of reinforced concrete structures. The non-steady state chloride migration coefficient - $\mathrm{D}_{\text {nssm, }}$ (apparent coefficient), obtained by the NT Build 492 method, can be a "marker" of concrete durability, while it is a simple laboratory test and is an input into mathematical models for predicting useful life. Thus, the aim of this work is to analyze the sensitivity of $\mathrm{D}_{\text {nssm }}$ in concrete without mineral additions and with the intervention of three strength classes (MPa), namely C45, C30 and C25, which correspond to the water/cement ratio (w/c) 0.40; 0.55 and 0.70 , respectively. As expected result, there was a correlation $\left(R^{2}=0.99\right)$ between the response parameter $D_{n s s m}$ and the variable w/c ratio or strength class at the levels studied. By changing the w/c ratio from 0.70 to 0.55 from 0.70 to $0.40, \mathrm{D}_{\text {nssm }}$ reduced by $41 \%$ and $61 \%$, respectively. The results were discussed based on the microstructure of concrete, statistical analysis and the migration coefficient, as a parameter of durability.

Keywords: Chloride; Migration; Diffusion; NT Build 492; Durability parameter.

\section{RESUMEN}

Los parámetros de durabilidad pueden ser herramientas útiles e interesantes en un enfoque basado en la ciencia de datos y en el modelado de rendimiento de campo de estructuras de hormigón armado. El coeficiente de migración de cloruro en estado no estacionario - $\mathrm{D}_{\mathrm{nssm}}$, (coeficiente aparente), obtenido por el método NT Build 492, puede ser un marcador de la durabilidad del concreto, mientras que es una prueba de laboratorio simple y es una entrada en modelos matemáticos para predecir la vida útil. Así, el objetivo de este trabajo es analizar la sensibilidad del $\mathrm{D}_{\mathrm{nssm}}$ en hormigones sin adiciones minerales y con la intervención de tres clases de resistencia (MPa), a saber, C45, C30 y C25, que corresponden a las relaciones agua / cemento (w/c) 0,40; 0,55 y 0,70 , respectivamente. Como resultado esperado, hubo una correlación $\left(\mathrm{R}^{2}=0,99\right)$ entre el parámetro de respuesta $D_{n s s m}$ y la variable relación a/c o clase de resistencia en los niveles estudiados. Al cambiar la relación a / c de 0,70 a 0,55 de 0,70 a 0,40 , el $\mathrm{D}_{\text {nssm }}$ se redujo en un $41 \%$ y un $61 \%$, respectivamente. Los resultados se discutieron con base en la microestructura del hormigón, el análisis estadístico y el coeficiente de migración, como parámetro de durabilidad.

Palabras clave: Cloruros, migración, difusión, NT Build 492; Parámetro de durabilidad.

\section{INTRODUÇÃO}

Dentre os problemas relacionados à construção civil, a corrosão das armaduras no concreto tem destaque devido ao grande número de casos, riscos estruturais envolvidos e elevado custo de recuperação e de manutenção (Cascudo, 1997). A Associação Nacional de Engenheiros de Corrosão dos Estados Unidos (National Association of Corrosion Engineers - NACE) estima que os custos globais em decorrência da corrosão, considerando diversos setores como agricultura, indústria e serviços foram de 2,5 trilhões de dólares no ano de 2013. Este número equivale a 3,4\% do produto interno bruto (PIB) mundial naquele ano (Koch et al., 2016). Em estudo mais recente na Suíça (Yilmaz and Angst, 2020), os custos anuais diretos de corrosão em pontes rodoviárias foram estimados em $0,08 \%$ do PIB do país.

Um dos principais agentes agressivos que ocasionam a corrosão das armaduras em estruturas de concreto é o íon cloro (cloreto), presente em ambientes marinhos. Assim, estudar os mecanismos de transporte de íons agressivos envolvidos e o comportamento da estrutura devido a esse e outros mecanismos de degradação são importantes para uma abordagem baseada no desempenho e para previsão da vida útil. Adicionalmente, nesta abordagem de desempenho, os parâmetros e 
marcadores de durabilidade são ferramentas importantes para compreender a durabilidade do concreto (Baroghel-Bouny et al., 2014a, b). A Associação Francesa de Engenharia Civil (Association Française de Génie Civil - AFGC) indica que o coeficiente de difusão (aparente ou real) de cloretos é um dos indicadores de durabilidade gerais do concreto (AFGC, 2004).

Dessa forma, um dos ensaios interessantes para indicar a resistência do concreto à penetração de cloretos é o ensaio da NT Build 492 ((NORDEST,1999), por meio da determinação do coeficiente de migração em estado não-estacionário (Noushini et al., 2021). Esse ensaio é baseado principalmente em estudos do pesquisador Tang (Tang and Nilsson, 1993; Tang and Sorensen, 1998) e é de curta duração, sendo inclusive utilizado por algumas normas e códigos como um dos parâmetros de entrada em modelos preditivos de iniciação da corrosão (Marques et al., 2012; fib 2006 e DuraCrete 2000).

Este trabalho tem como objetivo analisar as variações do coeficiente de migração de cloretos, a partir do método proposto pela norma NT Build 492 (NORDTEST, 1999), em concretos sem adições minerais, observando-se a interveniência da classe de resistência (MPa) (C45, C30 e C25), as quais correspondem as relações água/aglomerante $(\mathrm{a} / \mathrm{c}) 0,40 ; 0,55$ e 0,70 , respectivamente). Destaca-se que os níveis desta variável foram escolhidos, baseando-se em pesquisas anteriores ${ }^{2} \mathrm{e}$ nas classes usuais de concretos, produzidos comercialmente na cidade de Goiânia/GO e utilizados por construtoras em obras de construção civil. Além disso, o nível a/c de 0,55 está de acordo com a ABNT NBR 6118 (ABNT, 2014) que prescreve uma relação água/aglomerante menor ou igual a 0,55 para ambientes marinhos (classe de agressividade ambiental III - forte).

A contribuição deste artigo está na possibilidade de o coeficiente de migração em estado não estacionário apresentar-se como um parâmetro de durabilidade do concreto armado, por meio da correlação deste coeficiente com a classe de resistência e a relação água/aglomerante de concretos sem adições minerais.

\section{MATERIAIS E MÉTODOS}

\subsection{Materiais}

Para a produção dos concretos, foram utilizados os seguintes materiais:

- cimento Portland CP-V;

- areia natural quartzosa de leito de rio, como agregado miúdo;

- brita de rocha granítica, como agregado graúdo;

- água e aditivo superplastificante à base de éter policarboxílico modificado.

\subsection{Dosagem e caracterização do concreto fresco}

A Tabela 1 apresenta o a dosagem e as propriedades no estado fresco dos concretos estudados. Optou-se, nesse trabalho, por estudar concretos sem adições minerais.

Tabela 1. Dosagem dos concretos estudados e suas características no estado fresco.

\begin{tabular}{|l|l|l|l|l|l|l|l|l|}
\hline & & & & & Abatimento & & & Resistência à compressão \\
\hline
\end{tabular}

\footnotetext{
2 Pesquisas do GeDur (Grupo de Estudos em Durabilidade) do PPG-GECON/UFG entre os anos de 2001 e 2019 (em destaque: Carasek, Cascudo, 2001; Castro, 2003; Oliveira, 2007; Oliveira e Cascudo,2018; Oliveira, 2019, dentre outros estudos do grupo).
} 


\begin{tabular}{|c|c|c|c|c|c|c|c|c|c|c|}
\hline $\begin{array}{c}\text { Tipo de } \\
\text { concreto }\end{array}$ & $\begin{array}{l}\text { Rel. } \\
\text { a/c }\end{array}$ & $\begin{array}{c}\text { Traço em } \\
\text { massa } \\
\text { de cimento } \\
\text { (c: a: b) }\end{array}$ & $\begin{array}{c}\text { Consumo } \\
\text { de } \\
\text { cimento } \\
\left(\mathrm{kg} / \mathrm{m}^{3}\right)\end{array}$ & $\begin{array}{c}\text { Teor } \\
\text { de } \\
\text { Aditivo } \\
\text { (\%) }\end{array}$ & $(\mathbf{m m})$ & $\begin{array}{c}\text { Teor } \\
\text { de } \\
\text { ar } \\
(\%)\end{array}$ & $\begin{array}{c}\rho_{c}{ }^{*} \\
\left(\mathrm{~kg} / \mathrm{m}^{3}\right)\end{array}$ & $\begin{array}{l}\text { Valor } \\
\text { médio } \\
\text { (MPa) }\end{array}$ & $\begin{array}{l}C V^{*} \\
(\%)\end{array}$ & $\begin{array}{l}\text { Classe de } \\
\text { resistência }\end{array}$ \\
\hline C 45 & 0,40 & $1: 1,78: 2,10$ & 440 & 0,44 & 150 & 2,7 & 2113 & 43,9 & 3,0 & C 45 \\
\hline C 30 & 0,55 & $1: 2,57: 2,81$ & 330 & 0,35 & 155 & 3,3 & 2138 & 30,3 & 5,0 & C 30 \\
\hline C 25 & 0,70 & $1: 3,49: 3,67$ & 262 & 0,70 & 145 & 2,9 & 2088 & 24,2 & 4,0 & C 25 \\
\hline
\end{tabular}

* Massa específica do concreto no estado fresco

Foram moldados corpos de prova cilindros (diâmetro $10 \mathrm{~cm}$ e altura $20 \mathrm{~cm}$ ) com repetição de 3 amostras para cada condição de estudo. Quanto ao procedimento de cura, após a moldagem e durante as primeiras 48 horas, os corpos de prova (CPs) foram mantidos nas fôrmas metálicas no ambiente de laboratório e cobertos com camadas de filme plástico. Após esse período, os CPs foram desmoldados, identificados e mantidos em cura submersa (água saturada com cal) até a data de 28 dias de idade. Nesta data, foram realizados os ensaios de migração de cloretos pelo método NT BUILD 492 (NORDTEST, 1999).

\subsection{Ensaio de migração de cloretos}

A Figura 1 apresenta de forma esquemática as 5 etapas do ensaio de migração de cloretos pela norma NT Build 492 (NORDTEST, 1999).

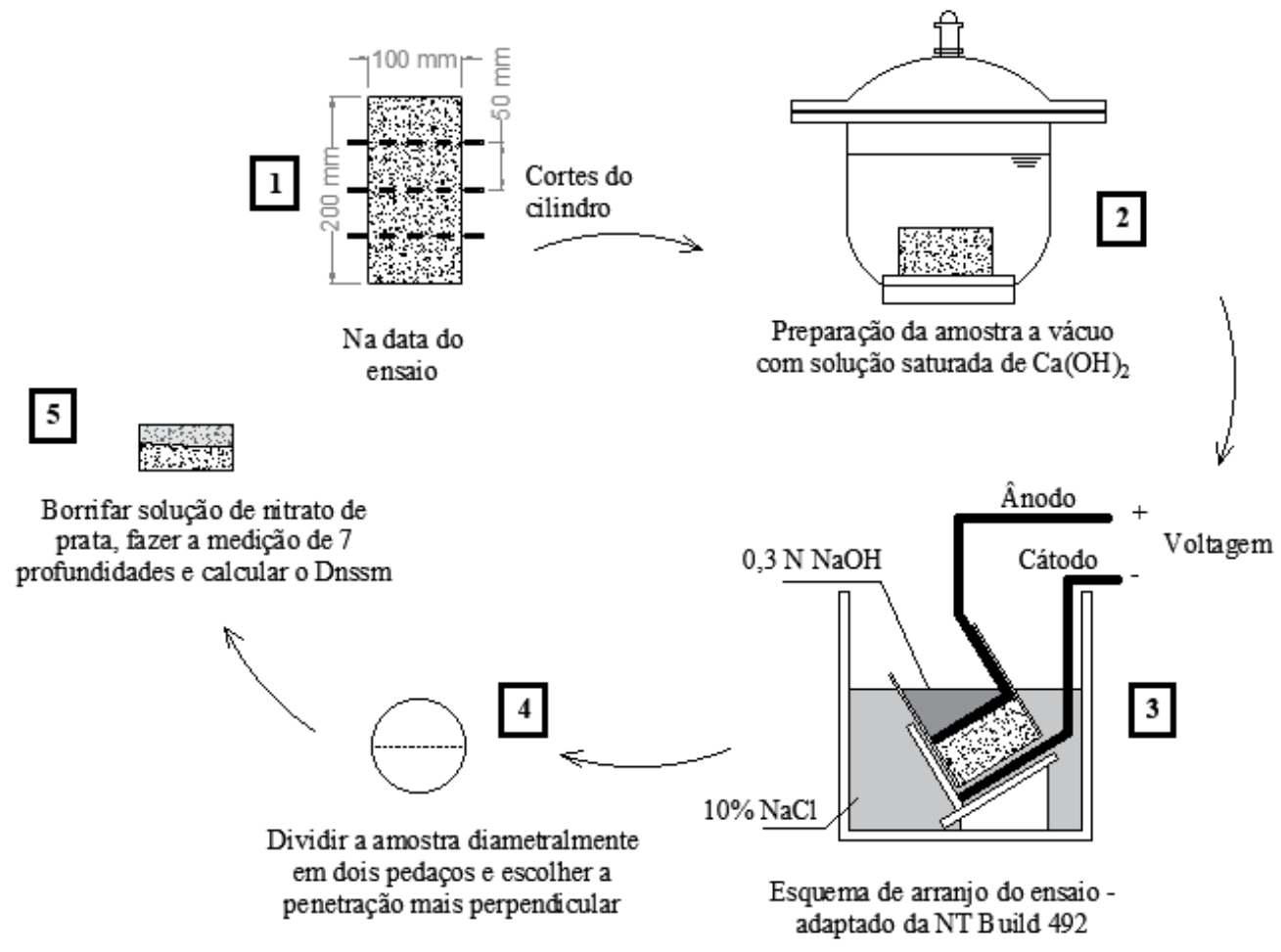

Figura 1. Desenho esquemático das etapas (1 a 5) do ensaio NT Build 492 (NORDTEST, 1999).

${ }^{3}$ ABNT NBR 8953:2015 - Concreto para fins estruturais. 
Os testes foram realizados com CPs de dimensões nominais de $100 \mathrm{~mm}$ de diâmetro e $50 \pm 2 \mathrm{~mm}$ de espessura, extraídos das amostras cilíndricos de $10 \mathrm{~cm}$ de diâmetro e $20 \mathrm{~cm}$ de altura. Os CPs foram colocados em vácuo por três horas (Figura 2a), seguido de saturação por $19 \pm 2$ horas em solução saturada de hidróxido de cálcio $\left[\mathrm{Ca}(\mathrm{OH})_{2}\right]$. Na configuração experimental do ensaio, cada $\mathrm{CP}$ foi envolvido por luva de borracha e abraçadeira. Também foram vedados com silicone acético para impedir a passagem da solução. A Figura $2 b$ apresenta a configuração experimental do ensaio.

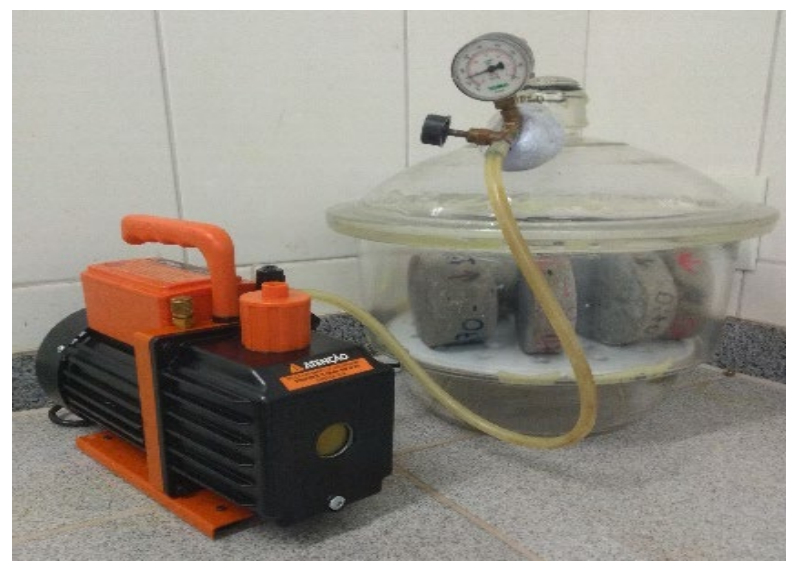

(a)

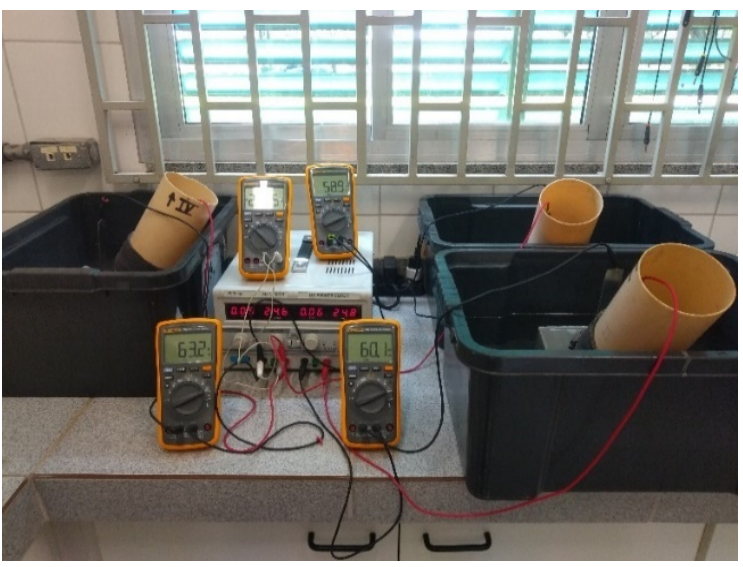

(b)

Figura 2. Ensaio NT Build 492 (1999): (a) amostras em vácuo no dessecador e (b) configuração geral experimental do ensaio com corpos de prova, multímetros e fonte elétrica.

Em resumo, este ensaio de migração consiste na aplicação de voltagem, ajustada conforme leitura inicial da corrente, para fazer os cloretos migrarem da solução do cátodo (12 litros de solução 10\% em massa de $\mathrm{NaCl}$ ) rica em cloretos para a solução do ânodo (300 ml de solução $0,3 \mathrm{M}-0,3 \mathrm{~mol} / \mathrm{L}$, aproximadamente $12 \mathrm{~g} / \mathrm{l}$ de $\mathrm{NaOH}$ ).

Ao fim dos ensaios, os CPs foram divididos em duas partes para medida da profundidade de penetração de cloretos $(\mathrm{mm})$, por meio da aspersão de solução a $0,1 \mathrm{M}$ de nitrato de prata $\left(\mathrm{AgNO}_{3}\right)$. Todos os reagentes químicos utilizados tiveram uma mesma procedência e foram PA (para análise). Para cada amostra testada, foram realizadas sete medidas da profundidade de penetração.

O coeficiente de difusão em estado não estacionário - $\mathrm{D}_{\text {nssm }}$ (ou coeficiente de difusão aparente) foi calculado a partir da profundidade de penetração de cloretos $(\mathrm{mm})$, das três amostras ensaiadas simultaneamente, do mesmo tipo e idade de concreto, pela Equação (1) simplificada.

$$
\mathrm{D}_{\mathrm{nssm}}=\frac{0,0239(273+\mathrm{T}) \mathrm{L}}{(\mathrm{U}-2) \mathrm{t}}\left(\mathrm{x}_{\mathrm{d}}-0,0238 \sqrt{\frac{(273+\mathrm{T}) \mathrm{L} \mathrm{x}_{\mathrm{d}}}{\mathrm{U}-2}}\right)
$$

Em que:

$\mathrm{D}_{\mathrm{nssm}}=$ coeficiente de migração em estado não estacionário $\left[\mathrm{x} 10^{-12} \mathrm{~m}^{2} / \mathrm{s}\right]$;

$\mathrm{U}=$ valor absoluto da voltagem aplicada $[\mathrm{V}]$;

$\mathrm{T}=$ média das temperaturas inicial e final da solução anódica $\left[{ }^{\circ} \mathrm{C}\right]$;

$\mathrm{L}=$ espessura da amostra [mm], média obtida de três medidas; 
$\mathrm{X}_{\mathrm{d}}=$ valor médio da profundidade de penetração [mm], obtido de sete medidas e

$\mathrm{t}=$ tempo de duração do ensaio [horas]

\section{RESULTADOS E DISCUSSÕES}

A Tabela 2 apresenta os resultados dos ensaios de migração de cloretos dos concretos estudados.

Tabela 2. Resultados do ensaio NT Build 492 (NORDTEST, 1999) aos 28 dias de idade.

\begin{tabular}{|c|c|c|c|c|c|}
\hline \multicolumn{2}{|c|}{$\begin{array}{l}\text { Tipo de } \\
\text { concreto }\end{array}$} & \multicolumn{2}{|c|}{$\begin{array}{l}\text { Penetração média } \\
\text { de cloretos }\end{array}$} & \multicolumn{2}{|c|}{$\begin{array}{l}\text { Coeficiente de difusão em } \\
\text { estado não estacionário - } \mathrm{D}_{\text {nssm }}\end{array}$} \\
\hline $\begin{array}{l}\text { Classe de } \\
\text { resistência }\end{array}$ & $\begin{array}{l}\text { Relação } \\
\text { a/c }\end{array}$ & $\begin{array}{l}\text { Valor } \\
\text { médio } \\
(\mathrm{mm})\end{array}$ & $\begin{array}{l}\mathrm{CV}^{*} \\
(\%)\end{array}$ & $\begin{array}{l}\text { Valor } \\
\text { médio } \\
\left(10^{-12} \mathrm{~m}^{2} / \mathrm{s}\right)\end{array}$ & $\begin{array}{l}\mathrm{CV}^{*} \\
(\%)\end{array}$ \\
\hline $\mathrm{C} 45$ & 0,40 & 23,40 & 4,0 & 10,88 & 4,0 \\
\hline C30 & 0,55 & 29,11 & 3,0 & 16,60 & 3,0 \\
\hline $\mathrm{C} 25$ & 0,70 & 48,28 & 2,0 & 27,94 & 3,0 \\
\hline
\end{tabular}

*CV (\%) é o coeficiente de variação dos resultados obtidos.

Da Tabela 2 é possível observar, como esperado e como resultado geral, que à medida que se aumenta a classe de resistência (e a relação a/c reduz), $\mathrm{D}_{\mathrm{nssm}}$ médio é reduzido. Dessa forma, com a redução da relação a/c de 0,70 para 0,55 , houve uma redução de $41 \%$ no coeficiente de migração e de 0,70 para 0,40 , uma diminuição de $61 \%$ com incremento nas classes de resistência de C25 para C30 e C25 para C45, respectivamente. Cabe ressaltar que em alguns pontos da medição do concreto de relação a/ac 0,70, a frente de migração de cloretos atingiu toda a espessura da amostra. Neste caso, a leitura de penetração de cloretos foi admitida como a própria espessura e apresentou o maior valor dentre os concretos estudados.

Salienta-se que os valores obtidos de $\mathrm{D}_{\text {nssm }}$ foram próximos de alguns dados da literatura. Hasholt and Jensen (2015) obtiveram um coeficiente de migração de aproximadamente $10 \times 10^{-12} \mathrm{~m}^{2} / \mathrm{s}$ para concreto sem adições minerais e relação a/c 0,40. Outros autores, Liu et al. (2011) apresentaram ao resultado de $8,8 \times 10^{-12} \mathrm{~m}^{2} / \mathrm{s}$ para uma relação a/c 0,38 e $19,1 \times 10^{-12} \mathrm{~m}^{2} / \mathrm{s}$ para uma relação a/c

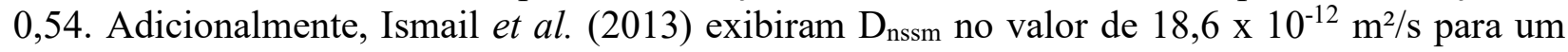
concreto com relação a/c 0,52. Outros autores (Luping, Nilsson and Bashee, 2012) relatam média de resultados de comparação inter laboratorial (6 laboratórios) pelo método da NT BUILD 492 para concretos classe $\mathrm{C} 45$ sem adições minerais de $18,6 \times 10^{12} \mathrm{~m}^{2} / \mathrm{s}$ com repetibilidade média de $7,5 \%$ de coeficiente de variação e de reprodutibilidade média de $16,2 \%$.

A Figura 3 exibe gráficos de correlação entre a relação a/c e o $D_{n s s m}$ e penetração de cloretos nos concretos estudados.
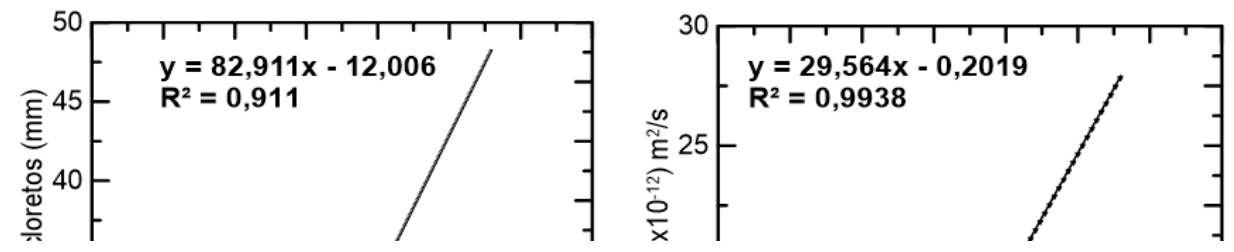

XVI Congreso Latinoamericano de Patología de la Construcción 
Figura 3. Correlação entre relação a/c versus (a) penetração de cloretos (mm) e (b) $\mathrm{D}_{\text {nssm }}$ médio $\left(\mathrm{x} 10^{-12} \mathrm{~m}^{2} / \mathrm{s}\right)$.

A Figura 3 ilustra a boa correlação e interação entre a variável (relação a/c) e a propriedade ( $\left.D_{\text {nssm }}\right)$ e a relação a/c e a penetração de cloretos nas amostras de concreto nos níveis das variáveis estudadas. $\mathrm{O} \mathrm{D}_{\text {nssm }}$ apresentou-se um parâmetro sensível para as diferenças microestruturais nos concretos em termos de porosidade e mecanismos de transporte. Esse comportamento já era esperado e concorda com a literatura de que altas relações a/c (e menores classes de resistências mecânicas - C25), comparativamente a relações a/c mais baixas, propiciam concretos com maior porosidade e permeabilidade, permitindo mais facilmente a entrada de cloretos. Com a entrada de cloretos facilitada, ocorre ruptura da película passivadora que protege o aço e a corrosão inicia-se. Isso na prática, se reflete em potenciais de corrosão das armaduras mais eletronegativos, redução das resistividades elétricas do concreto, redução dos valores de resistência de polarização do aço e aumento das taxas de corrosão (OLIVEIRA; CASCUDO, 2018).

Os valores de $\mathrm{D}_{\text {nssm }}$ foram condicionados, testados em relação ao espúrios (teste de Dixon ASTM 178-08) e submetidos à análise de variâncias (ANOVA one-way). A Tabela 3 apresenta os resultados obtidos para um nível de confiança de $95 \%$.

Tabela 3. Resultados da análise de variância (ANOVA) realizada com dados de $\mathrm{D}_{\mathrm{nss}}$,

\begin{tabular}{|c|c|c|c|c|c|l|}
\hline Efeito & $\begin{array}{c}\text { Soma dos } \\
\text { quadrados }\end{array}$ & $\begin{array}{c}\text { Graus de } \\
\text { Liberdade }\end{array}$ & $\begin{array}{c}\text { Média dos } \\
\text { Quadrados }\end{array}$ & F calculado & P-Valor & Resultado \\
\hline Modelo & 3071,10 & 1 & 3071,10 & 8187,90 & 0,0000 & Significativo \\
\hline Relação a/c & 452,05 & 2 & 226,03 & 602,63 & 0,0000 & Significativo \\
\hline Erro & 2,25 & 6 & 0,38 & & & \\
\hline Total & 454,30 & 8 & - & - & - \\
\hline \multicolumn{7}{|c|}{$\begin{array}{c}\mathrm{R}^{2} \bmod =0,99(\text { coeficiente de determinação do modelo) } \\
\text { Rmod }=1,00(\text { coeficiente de correlação do modelo) }\end{array}$} \\
\hline
\end{tabular}

A Tabela 3 apresentou que o modelo fatorial é significativo, uma vez que o valor- $\mathrm{P}^{4}$ é menor que $\alpha(0,05)$. Ao mesmo tempo, o valor resultante de $\mathrm{R}^{2}{ }_{\text {mod }}$ foi de 0,99 , o que significa dizer que $99 \%$ da variação total dos dados estudados é explicada pelo modelo obtido. Há claramente uma relação entre a variável quantitativa $(\mathrm{a} / \mathrm{c})$ e a propriedade avaliada $\left(\mathrm{D}_{\mathrm{nssm}}\right)$. A Tabela 3 também indicou

\footnotetext{
${ }^{4}$ Se o p-valor ou valor-P é menor que o nível de significância $(\alpha=0,05)$, rejeitamos a hipótese nula de que todos os concretos nos níveis de relação a/c adotados são iguais.
} 
diferenças entre os $\mathrm{D}_{\text {nssm }}$ dos concretos nas relações a/c estudadas a um nível de confiança de $95 \%$ e, por isso, realizou-se uma comparação múltiplas de médias pelo método de Duncan. O resultado é apresentado na Figura 4.

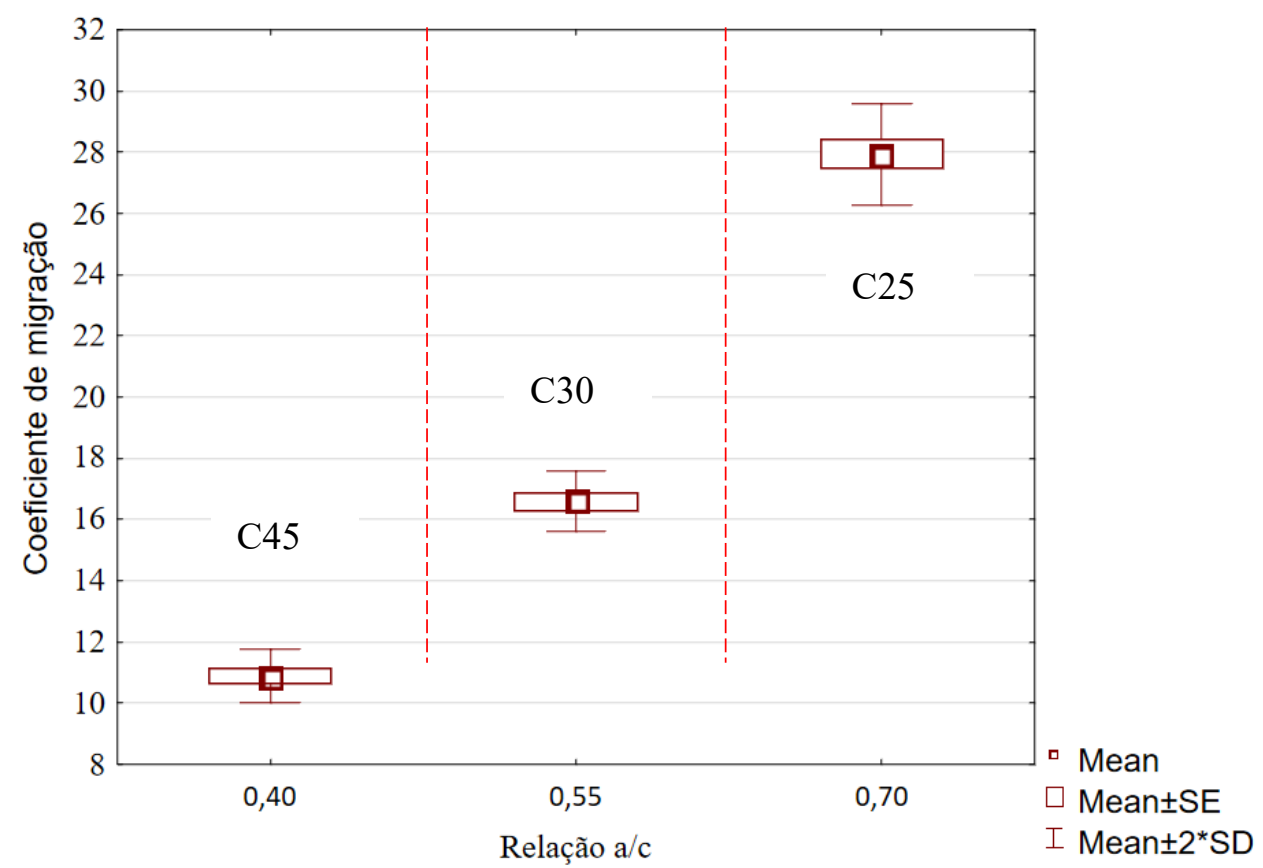

Figura 4. Valores médios de globais de $\mathrm{D}_{\text {nssm }}\left(\times 10^{-12} \mathrm{~m}^{2} / \mathrm{s}\right)$ dos concretos estudados por relação a/c com seus intervalos de confiança de $95 \%$ e seus desvios padrões. A linha tracejada vertical define os grupos (1-3) que diferem significativamente.

A Figura 4 indica o resultado da comparação múltiplas de médias, o qual resultou em 3 grupos estatísticos distintos (as linhas tracejadas verticais definem os grupos que diferem significativamente entre si), isto é, os concretos nos níveis de relações a/c estudadas $(0,40 ; 0,55$ e $0,70)$ são estatistamente diferentes. Além disso, cabe o comentário que a análise estatística validou e expressou diferenças que já observamos sob o ponto de vista técnico, prático e microscópico destes concretos. Além disso, financeiramente e sob a óptica de engenharia tecnológica, os concretos estudados também são diferentes.

Por fim, cabe destacar que menores valores de $\mathrm{D}_{\text {nssm, }}$, expressos nas menores relações a/c e maiores classes de resistência denotam maior durabilidade às estruturas de concreto armado e maior vida útil, mesmo em ambientes agressivos, sobretudo contendo cloretos. Paralelamente, concretos duráveis apresentam taxas mais baixas de corrosão de armaduras, valores de potenciais de corrosão mais eletropositivos e maiores resistências de polarização do aço (Oliveira e Cascudo, 2018).

\section{CONCLUSÃO}

Por meio dos resultados apresentados, pode-se concluir que:

- a resistência do concreto à penetração de cloretos teve boa correspondência com a relação a/c $\left(\mathrm{R}^{2}=0,99\right)$;

- a medida que a relação a/c reduziu de 0,70 para 0,40 e, ao contrário a classe de resistência aumentou de $\mathrm{C} 25$ para $\mathrm{C} 45$, o $\mathrm{D}_{\text {nssm }}$ médio reduziu, como consequência das alterações 
microscópicas na matriz hidratada em termos de porosidade e zonas de interface agregado matriz. Isto também se reflete em termos de mecanismos de transportes na matriz hidratada;

- concretos de relação a/c 0,70; 0,55 e 0,40 com respectivas classes de resistência C25, C30 e C45 apresentaram $D_{\text {nssm }}$ médio de $27,94 \times 10^{-12} ; 16,60 \times 10^{-12}$ e $10,88 \times 10^{-12} \mathrm{~m}^{2} / \mathrm{s}$ nesta ordem com coeficiente de variação máximo de $4 \%$ entre os resultados para cada condição de estudo.

- a alteração da relação a/c de 0,70 para 0,55 de 0,70 para 0,40 , reduziu $\mathrm{D}_{\text {nssm }}$ médio em $41 \%$ e $61 \%$;

- a análise estatística evidenciou a formação de 3 grupos estatísticos distintos por relação a/c estudada.

- o coeficiente de migração apresentou-se ser um parâmetro sensível e com validade em termos de durabilidade do concreto armado, sobretudo em estruturas situadas em ambientes contendo cloretos.

\section{AGRADECIMENTO}

Este trabalho faz parte do projeto de P\&D 0394-1504-2015, regulamentado pela Agência Nacional de Energia Elétrica - ANEEL, desenvolvido pela Eletrobras FURNAS e FUNAPE/UFG/EECA/LABITECC.

\section{REFERÊNCIAS}

AFGC. Association Francaise de Genie Civil. (2014). Conception des bétons pour une durée de vie donnée des ouvrages. Documents scientifiques et techniques.

American Society For Testing of Materials. ASTM E178-08, Standard Practice for Dealing With Outlying Observations, ASTM International, West Conshohocken, PA, 2008, www.astm.org.

Associação Brasileira de Normas Técnicas. (2014). NBR 6118: Projeto de Estruturas de Concreto - Procedimento. Rio de Janeiro.

Associação Brasileira de Normas Técnicas. (2015). NBR 8953: Concreto para fins estruturais Classificação pela massa específica, por grupos de resistência e consistência. Rio de Janeiro.

Associação Brasileira de Normas Técnicas. (2002). NBR NM 47: Concreto - Determinação do teor de ar em concreto fresco - Método pressométrico. Rio de Janeiro.

Associação Brasileira de Normas Técnicas. (1998). NBR NM 67: Concreto - Determinação da consistência pelo abatimento do tronco de cone. Rio de Janeiro.

Baroghel-Bouny, V., Capra, B., Laurens, S. (2014a), “A durabilidade das armaduras e do concreto de cobrimento". In: Olliver, J.-P.; Vichot, A. (Ed) Durabilidade do Concreto: Bases científicas para a formulação de concretos duráveis de acordo como ambiente. Tradução: Cascudo, O., Carasek, H. IBRACON, São Paulo, p. 255-326.

Baroghel-Bouny, V., Cussigh, F., Rougeau, P. (2014b), "Durabilidade dos concretos a partir da abordagem do desempenho”. In: Olliver, J.-P.; Vichot, A. (Ed) Durabilidade do Concreto: Bases científicas para a formulação de concretos duráveis de acordo como ambiente. Tradução: Cascudo, O., Carasek, H. IBRACON, São Paulo, p 237-254. 
Carasek, H.; Cascudo, O. (2001). Projeto integrado: características do concreto de cobrimento e o seu papel na durabilidade das estruturas de concreto armado, Goiânia (projeto de pesquisa).

Cascudo, O. (1997), "O controle da corrosão de armaduras em concreto: inspeção e técnicas eletroquímicas”. Pini, 1. ed., São Paulo, p. 237.

Castro, A. (2003), "Influência das adições minerais na durabilidade do concreto sujeito à carbonatação”, Dissertação (Mestrado em Engenharia Civil), Universidade Federal de Goiás, p. 215.

DuraCrete (2000). "DuraCrete Final Technical Report, General Guide-lines for Durability Design and Redesign”, Document BE95-1347/R17, The European Union -Brite EuRam III, Netherlands.

fib (2006) International Federation for Structural Concrete: fib Model Code for Service Life Design. Bulletin No 34., Lausanne, Switzerland.

Hasholt, M. T., Jensen, O. M. (2015), "Chloride migration in concrete with superabsorbent polymers". Cement and Concrete Composites, 55: 290-297. http://dx.doi.org/10.1016/j.cemconcomp.2014.09.023

Ismail, I., Bernal, S. A., Provis, J. L., San Nicolas, R., Brice, D. G., Kilcullen, A. R., . Van Deventer, J. S. J. (2013). Influence of fly ash on the water and chloride permeability of alkali-activated slag mortars and concretes. Construction and Building Materials, 48: 1187-1201. http://dx.doi.org/10.1016/j.conbuildmat.2013.07.106

Koch, G., Varney, J., Thompson, N., Moghissi, O., Gould, M., Payer, J. (2016), International measures of prevention, application, and economics of corrosion technologies study. National Association of Corrosion Engineers - NACE International, p. 216.

Liu, X., Chia, K. S., Zhang, M. H. (2011), Water absorption, permeability, and resistance to chloride-ion penetration of lightweight aggregate concrete. Construction and Building Materials, 25(1): 335-343. http://dx.doi.org/10.1016/j.conbuildmat.2010.06.020

Luping, T., Nilsson, L.-O., Bashee, M.P.A. (2012), "Resistance of Concrete to Chloride Ingress Resistance of Concrete to Chloride Ingress Testing and Modelling". RC Press Taylor \& Francis International, London. Standard Book Number-13: 978-0-203-88241-2 (eBook - PDF)

Marques, P. F., Costa, A., Lanata, F. (2012), Service life of RC structures: chloride induced corrosion: prescriptive versus performance-based methodologies. Materials and Structures, 45: 277-296. http://dx.doi.org/10.1617/s11527-011-9765-2

NORDTEST. (1999), N.T. Build 492: Concrete, mortar and cement-based repair materials: Chloride migration coefficient from non-steady-state migration experiments. Finland.

Noushini, A., Nguyen, Q. D., Castel, A. (2021), Assessing alkali-activated concrete performance in chloride environments using NT Build 492. Materials and Structures, 54 (2): 1-15, 2021, https://doi.org/10.1617/s11527-021-01652-7 
Oliveira, A. M. (2007), “Avaliação do desempenho de concretos com adições minerais quanto à corrosão das armaduras induzida por cloretos”. Dissertação (Mestrado em Engenharia Civil), Universidade Federal de Goiás, p. 255.

Oliveira, A. P. (2019), "Estudo de matrizes cimentícias ternárias contendo sílica ativa e nanossílica”. Dissertação (Mestrado em Construção Civil), Universidade Federal de Goiás, p. 145.

Oliveira, A. M., Cascudo, O. (2018), Effect of mineral additions incorporated in concrete on thermodynamic and kinetic parameters of chloride-induced reinforcement corrosion. Construction and Building Materials, 192: 467 - 477. https://doi.org/10.1016/j.conbuildmat.2018.10.100

Tang, L., Sørensen, H. E. (1998), Evaluation of the Rapid Test Methods for Chloride Diffusion Coefficient of Concrete, NORDTEST Project No. 1388-98, SP Report 1998:42, SP Swedish National Testing and Research Institute, Sweden.

Tang, L., Nilsson, L.-O. (1993), Rapid determination of the chloride diffusivity in concrete by applying an electric field. Materials Journal, 89 (1): 49-53.

Yilmaz, D., Angst, U. (2020). Custos relacionados à corrosão de estruturas de engenharia civil na rede rodoviária suíça (artigo em alemão). Beton-und Stahlbetonbau, 115 (6): 448-458. 